\title{
Association of prior antiplatelet agents with mortality in sepsis patients
}

\author{
Min-Juei Tsai ${ }^{1,2}$, Chia-Jen Shih ${ }^{2,4}$ and Yung-Tai Chen ${ }^{2,3^{*}}$
}

Platelet activation is not only an essential component of primary hemostasis but also plays a critical role in disease progression during sepsis. Sepsis involves inflammatory processes through releasing of several inflammatory mediators, such as interleukin (IL)-1 $\beta$, IL-8, monocyte chemotactic protein 1 , and tumor necrosis factor alpha [1], and then influences microvascular thrombosis formation and both innate and adaptive immunity [2]. Thus, attenuation of platelet activation is suggested to be one possible treatment option for sepsis in preclinical studies [1]. Some retrospective observational studies have also revealed that prehospital antiplatelet use was associated with decreased mortality rate in sepsis patients [3-6], but there is still a place for argument until studies provide strong evidence to support whether antiplatelet drugs are a possible adjuvant treatment choice in sepsis (Table 1).

In a recent article in Intensive Care Medicine, Dr. Wiewel and colleagues introduced a prospective observational cohort study to determine the association between pre-existing antiplatelet treatment and outcome of sepsis [16]. This study enrolled patients with strict diagnostic criteria of sepsis and reported that chronic antiplatelet treatment was not associated with the development of organ failure or shock during intensive care unit (ICU) stay or mortality after admission. It also disclosed no differences in 19 biomarkers between antiplatelet users and non-users during ICU stay. Although the robustness of this study is strengthened by well-defined sepsis diagnosis and documentation of relevant molecular biomarkers

\footnotetext{
*Correspondence: ytchen0117@gmail.com

${ }^{3}$ Department of Nephrology, Taipei City Hospital Heping Fuyou Branch, Taipei, Taiwan 112

Full author information is available at the end of the article

The corresponding authors have the right to grant on behalf of all authors and do grant on behalf of all authors a worldwide license to the publishers and its licensees in perpetuity.
}

as an indicator of host immune response during sepsis, several concerns should be clarified.

First, in the study by Wiewel et al. [16], the size of only 150 patients per group who remained after propensity score matching is too small relative to the expected size of population ( $>2000)$ to detect differences in both groups. The definite impact (neutral, harm, or benefit) of antiplatelet agents on sepsis may not be determined thoroughly in this study because it is underpowered. This is not the only negative report with the same concern about inadequate sample size. Valerio-Rojas et al. [8] investigated 651 sepsis patients with similar study design and reported insignificant association between antiplatelet treatment and mortality. However, they also addressed the limitation and concern about inadequate patient number and power. With an eye toward improving reliability, the number of patients should be expanded to reach sufficient power in the further studies.

Second, given lack of details of antiplatelet prescription (dose and duration) and unknown baseline biomarker levels prior to antiplatelet drugs, we could not exclude that this flaw may elicit an unexpected finding of insignificant difference of 19 biomarkers between antiplatelet users and non-users with sepsis, possibly as a result of antiplatelet drug resistance or inappropriate dosing. Besides, there are more than 170 different putative biomarkers linked to sepsis, but most of them serve as predictors rather than as therapeutic guidance [17]. Those biomarkers that were reported to be associated with aspirin administration in sepsis, such as inhibition of nuclear factor kappa B [18], production of nitric oxide, and production of lipoxin $[7,10]$, were not investigated by the current study. The lack of difference in 19 currently used biomarkers between antiplatelet users and non-users may therefore not represent a lack of benefit.

Moreover, the lack of data regarding timing of antiplatelet administration or discontinuation does not allow

\section{Springer}




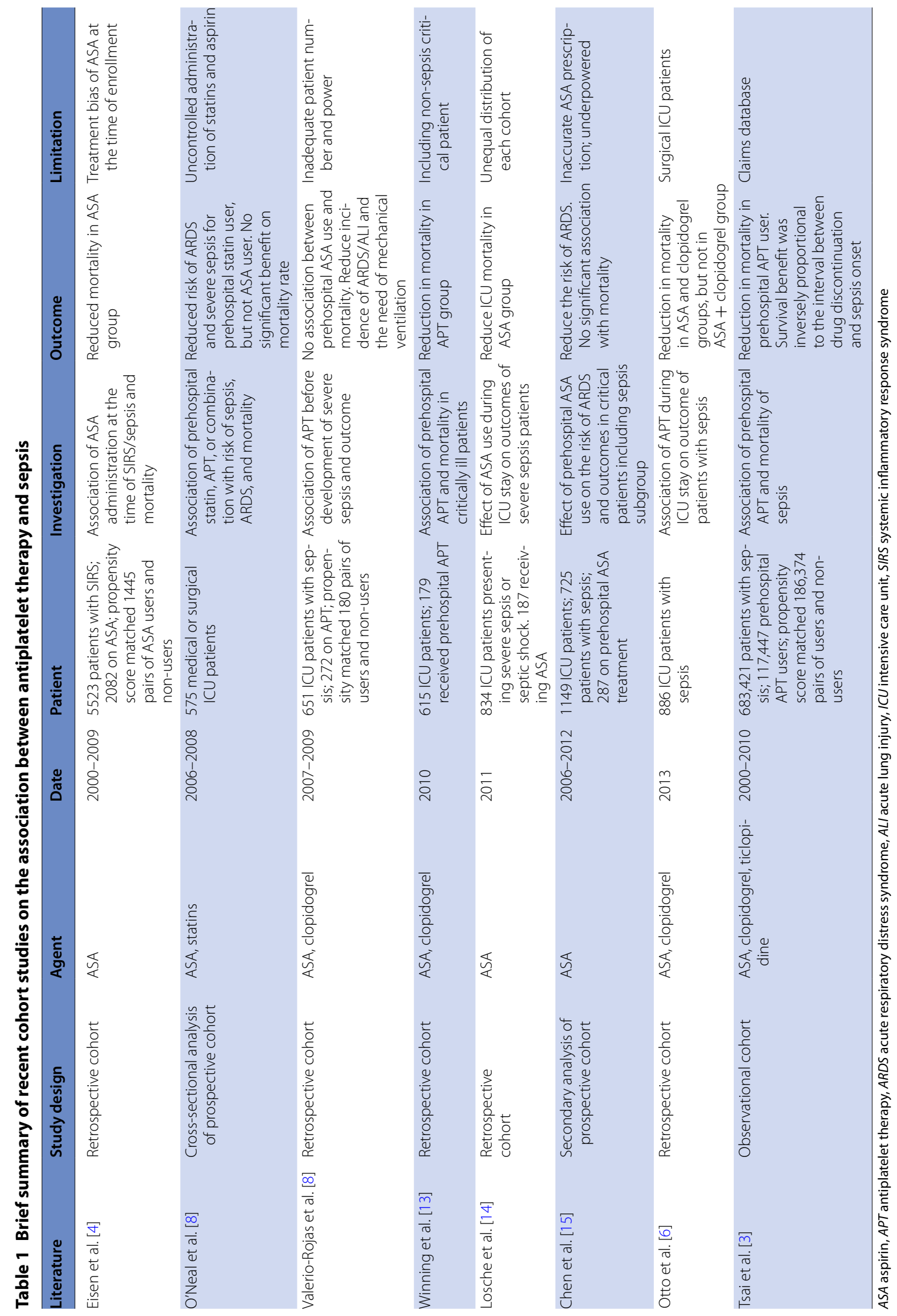


the authors to address the effect of these potential confounding variables on the outcomes. As we know, the inhibitory effect of aspirin or clopidogrel on platelet activation could last for about 1 week despite discontinuing these agents $[12,11]$. Our previous registry study also found that the benefit of prehospital use of antiplatelet drugs on sepsis outcomes was strongest in current users followed by recent users [3]. However, in the current study, the use of antiplatelet agents before enrollment was not clear. Besides, possible receipt of antiplatelet agents after ICU admission in non-antiplatelet users wound tend to bias the results to the null. Furthermore, only less than $50 \%$ of patients still had the antiplatelet drugs in the first 2 days of ICU admission. Therefore, early discontinuation of antiplatelet drugs prior to sepsis onset could also partially explain the neutral association in this study.

Although there are some limitations, this prospective observational cohort study introduced by Dr. Wiewel and colleagues is still very valuable. It is the first prospective study in this field and enrolled sepsis patients according to well-defined clinical evidence rather than database or chart review. It also performed meticulous propensity matching, including disease severity by APACHE score, SOFA score, organ failure, and shock, which is lacking in most previous investigations and may causing significant confounding.

Given the conflicting results and inherent limitations from observational studies, only randomized controlled trials aimed at exploring the potential roles of antiplatelet agents as adjuvant treatment in sepsis or preventive agents to reduce disease severity will integrate the best evidence into clinical care of sepsis patients. There are currently at least two ongoing relevant clinical trials. The first trial is "Aspirin for the Treatment of Sepsis" (NCT01784159) which will investigate the beneficial effect of aspirin treatment for 7 days on organ dysfunction and duration of ventilation in severe sepsis patients. Another one is "Aspirin to Inhibit Sepsis" (ANTISEPSIS, ACTRN12613000349741) which will assess the effect of daily aspirin treatment on the mortality and admission to ICU for sepsis. Overall, we suggest that clinicians must be cautious in prescribing routine prophylactic antiplatelet drugs for sepsis prevention of treatment in view of further cost-effectiveness and harm-benefit analyses with hard evidence.

\footnotetext{
Author details

${ }^{1}$ Department of Medicine, Chang-Hua Hospital, Ministry of Health and Welfare, Changhua, Taiwan 112. ${ }^{2}$ National Yang-Ming University School of Medicine, Taipei, Taiwan. ${ }^{3}$ Department of Nephrology, Taipei City Hospital Heping Fuyou Branch, Taipei, Taiwan 112. ${ }^{4}$ Deran Clinic, Yilan, Taiwan.
}

\section{Conflicts of interest}

The authors declare that they have no conflicts of interest.

\section{Funding sources}

none.

Received: 22 December 2015 Accepted: 12 January 2016 Published online: 12 February 2016

\section{References}

1. Toner P, McAuley DF, Shyamsundar M (2015) Aspirin as a potential treatment in sepsis or acute respiratory distress syndrome. Crit Care 19:374

2. de Stoppelaar SF, van't Veer C, van der Poll T (2014) The role of platelets in sepsis. Thromb Haemost 112:666-677

3. Tsai MJ, Ou SM, Shih CJ, Chao PW, Wang LF, Shih YN, Li SY, Kuo SC, Hsu YT, Chen YT (2015) Association of prior antiplatelet agents with mortality in sepsis patients: a nationwide population-based cohort study. Intensive Care Med 41:806-813

4. Eisen DP, Reid D, McBryde ES (2012) Acetyl salicylic acid usage and mortality in critically ill patients with the systemic inflammatory response syndrome and sepsis. Crit Care Med 40:1761-1767

5. Sossdorf M, Otto GP, Boettel J, Winning J, Losche W (2013) Benefit of lowdose aspirin and non-steroidal anti-inflammatory drugs in septic patients. Crit Care 17:402

6. Otto GP, Sossdorf M, Boettel J, Kabisch B, Breuel H, Winning J, Losche W (2013) Effects of low-dose acetylsalicylic acid and atherosclerotic vascular diseases on the outcome in patients with severe sepsis or septic shock. Platelets 24:480-485

7. O'Neal HR, Jr., Koyama T, Koehler EA, Siew E, Curtis BR, Fremont RD, May AK, Bernard GR, Ware LB (2011) Prehospital statin and aspirin use and the prevalence of severe sepsis and acute lung injury/acute respiratory distress syndrome. Crit Care Med 39:1343-1350

8. Valerio-Rojas JC, Jaffer IJ, Kor DJ, Gajic O, Cartin-Ceba R (2013) Outcomes of severe sepsis and septic shock patients on chronic antiplatelet treatment: a historical cohort study. Crit Care Res Pract 2013:782573

9. Winning J, Neumann J, Kohl M, Claus RA, Reinhart K, Bauer M, Losche W (2010) Antiplatelet drugs and outcome in mixed admissions to an intensive care unit. Crit Care Med 38:32-37

10. Losche W, Boettel J, Kabisch B, Winning J, Claus RA, Bauer M (2012) Do aspirin and other antiplatelet drugs reduce the mortality in critically ill patients? Thrombosis 2012:720254

11. Chen W, Janz DR, Bastarache JA, May AK, O'Neal HR Jr, Bernard GR, Ware $L B$ (2015) Prehospital aspirin use is associated with reduced risk of acute respiratory distress syndrome in critically ill patients: a propensity-adjusted analysis. Crit Care Med 43:801-807

12. Wiewel MA, de Stoppelaar SF, van Vught LA, Frencken JF, Hoogendijk AJ Klein Klouwenberg PMC, Horn J, Bonten MJ, Zwinderman AH, Cremer OL, Schultz MJ, van der Poll T, On behalf of the MARS Consortium (2015) Chronic antiplatelet therapy is not associated with alterations in the presentation, outcome or host response biomarkers during sepsis: a propensity-matched analysis. Intensive Care Med. doi:10.1007/ s00134-015-4171-9

13. Pierrakos C, Vincent JL (2010) Sepsis biomarkers: a review. Crit Care 14:R15

14. Weber C, Erl W, Pietsch A, Weber PC (1995) Aspirin inhibits nuclear factorkappa B mobilization and monocyte adhesion in stimulated human endothelial cells. Circulation 91:1914-1917

15. El Kebir D, Jozsef L, Pan W, Wang L, Petasis NA, Serhan CN, Filep JG (2009) 15-epi-lipoxin A4 inhibits myeloperoxidase signaling and enhances resolution of acute lung injury. Am J Respir Crit Care Med 180:311-319

16. Akinosoglou K, Alexopoulos D (2014) Use of antiplatelet agents in sepsis: a glimpse into the future. Thromb Res 133:131-138

17. Price MJ, Coleman JL, Steinhubl SR, Wong GB, Cannon CP, Teirstein PS (2006) Onset and offset of platelet inhibition after high-dose clopidogrel loading and standard daily therapy measured by a point-of-care assay in healthy volunteers. Am J Cardiol 98:681-684

18. Schror K (1997) Aspirin and platelets: the antiplatelet action of aspirin and its role in thrombosis treatment and prophylaxis. Semin Thromb Hemost 23:349-356 